\title{
RIR - Measurement and Use in Quantitative XRD
}

\author{
By Camden R. Hubbard \\ Ceramics Division, National Bureau of Standards, Gaithersburg, Maryland 20899 U.S.A. \\ Robert L. Snyder \\ New York State College of Ceramics, Alfred University, Alfred, New York 14802 U.S.A.
}

\begin{abstract}
The Reference Intensity Ratio (RIR) is a general, instrument-independent constant for use in quantitative phase analysis by the X-ray powder diffraction internal standard method. When the reference standard is corundum, RIR is known as I/I $I_{c}$; These constants are collected in the Powder Diffraction File (1987), can be calculated, and can be measured. Recommended methods for accurate measurement of RIR constants are presented, and methods of using these constants for quantitative analysis are discussed. The numerous, complex constants in Copeland and Bragg's method introduced to account for superimposed lines can be simply expressed in terms of RIR constants and relative intensities. This formalism also permits introduction of constraints and supplemental equations based on elemental analysis.
\end{abstract}

\section{Introduction}

Quantitative phase analysis by X-ray powder diffraction dates back to when Navias (1925) quantitatively determined the amount of mullite in fired ceramics, and to when Clark and Reynolds (1936) reported an internal standard method for mine dust analysis by film techniques. The development of the Geiger-counter diffractometer in 1945 led to increased possibilities; shortly thereafter Alexander and Klug (1948) presented the theoretical understanding for the absorption effects on diffraction intensities from a flat brickette of powder. Since then there have been numerous methods developed based on their basic equations. Methods applicable to a wide range of phases and samples include the addition of analyte or spiking method (Lennox, 1957), the intensity ratio method (Alexander and Klug, 1948; Smith, Snyder and Brownell, 1979) and the internal standard method (Klug and Alexander, 1974a). Formalisms have been established to permit inclusion of overlapping lines in the analysis (Copeland and Bragg, 1958). Unfortunately, far less effort has been placed on understanding the sample- and instrument-dependent limitations of quantitative phase analysis by $\mathrm{X}$-ray powder diffraction. Further, very little has been reported on combining quantitative elemental information with diffraction data to impiove the accuracy of quantitative phase analysis.

The internal standard method is amenable to very general use and is the only method for which calibration constants can be transferred from laboratory to laboratory. In addition, calibration constants can be calculated when detailed structural data are known (Hubbard, Evans and Smith, 1976). Following a recommendation by Visser and de Wolff (1964) the International Centre for Diffraction Data (ICDD) has been collecting a set of internal standard calibration constants, known as $I / I_{\text {corundum }}\left(I / I_{c}\right)$, for use in quantitative phase analysis. With the increased use of automated diffractometers and availability of software to simplify data measurement, quantitative XRD analysis has received renewed interest. To meet the needs of users the ICDD is exploring methods to add additional $\mathrm{I} / \mathrm{I}_{\mathrm{c}}$ values to the Powder Diffraction File (PDF)(1987). The I/ $I_{c}$ concept can be generalized into a Reference Intensity Ratio (RIR) method, as shown below, by replacing corundum with a general internal standard.

The purpose of this paper is to summarize the basis for quantitative analysis by RIR methods including how to measure RIR's and how to use these values in quantitative analysis.

\section{Basic Theory for Reference Intensity Ratios}

The intensity of diffraction line i from phase $\alpha$ from a flat plate is given by

$$
\mathrm{I}_{\mathrm{i} \alpha}=\frac{\mathrm{K}_{\mathrm{i} \alpha} \mathrm{X}_{\alpha}}{\rho_{\alpha}(\mu / \boldsymbol{\rho})_{\mathrm{m}}}
$$

where

$$
\begin{aligned}
& \mathrm{X}_{\alpha}=\text { weight fraction of phase } \alpha \\
& \rho_{\alpha}=\text { density of phase } \alpha \\
& (\mu / \rho)_{\mathrm{m}}=\text { mass absorption coefficient of the mixture }
\end{aligned}
$$

and where $\mathrm{K}_{\mathrm{i} \alpha}$, which is a constant for a given crystal structure $\alpha$, a diffraction line i, and a set of experimental conditions is:

$$
\begin{aligned}
& \mathrm{K}_{\mathrm{i} \alpha}=\frac{\mathrm{I}_{\mathrm{o}} \lambda^{3} \mathrm{e}^{4}}{32 \pi \mathrm{rm}^{2} \mathrm{c}^{4}} \times \frac{\mathrm{M}_{\mathrm{i}}}{2 \mathrm{~V}_{\alpha}^{2}}\left|\mathrm{~F}_{\mathrm{i} \alpha}\right| 2\left\{\frac{1+\cos ^{2} 2 \theta}{\sin ^{2} \theta \cos \theta}\right\}(2) \\
& \mathrm{I}_{\mathrm{o}}=\text { incident beam intensity } \\
& \mathrm{r}=\text { radius of the diffractometer (i.e., sample to } \\
& \lambda=\text { detector distance) } \\
& \lambda=\text { wavelength of the X-radiation } \\
& \mathrm{c}=\text { speed of light } \\
& \mathrm{e} \text { and } \mathrm{m}=\text { charge and mass of an electron } \\
& \mathrm{M}_{\mathrm{i}}=\text { multiplicity for reflection } \mathrm{i} \\
& \mathrm{V}_{\alpha}=\text { volume of the unit cell of phase } \alpha \\
& \mathrm{F}_{\mathrm{i} \alpha}=\text { structure factor }=\sum_{\mathrm{j}} \mathrm{f}_{\mathrm{j}} \mathrm{e}
\end{aligned}
$$

$\mathrm{h}, \mathrm{k}, \ell=$ Miller indices of line $\mathrm{i}$ of phase $\alpha$

$\mathrm{X}_{\mathrm{j}}, \mathrm{Y}_{\mathrm{j}}, \mathrm{Z}_{\mathrm{j}}=$ fractional coordinates for atom $\mathrm{j}$ of phase $\alpha$

$\mathrm{f}_{\mathrm{j}}=$ atomic scattering factor $\left[=\mathrm{f}_{\mathrm{j}}^{\mathrm{o}} \exp \left(-\mathrm{B}_{\mathrm{j}} \sin ^{2}(\theta) / \lambda^{2}\right)\right]$

$\mathrm{B}_{\mathrm{j}}=$ Debye-Waller temperature factor for atom $\mathrm{j}$ in phase $\alpha$

$\theta=$ Bragg diffraction angle

$\left(1+\cos ^{2} 2 \theta\right) /\left(\sin ^{2} \theta \cos \theta\right)=$ the Lorentz and Polarization corrections for a diffractometer

When we have more than two components present (whether they are known phases or not) and when the $\mu / \rho$ of the unknown cannot be computed (i.e., the chemical composition is not known) then we resort to the most general quantitative analysis technique - the internal standard method. This method applies only to powders, in that a known weight percent of a standard material must be homogeneously mixed with the sample of unknown phase composition. 
The internal standard method is based on elimination of the matrix absorption effects factor $(\mu / \rho)_{\mathrm{m}}$ in Equation (1) by forming the ratio $I_{i \alpha} / I_{j s}$, i.e. the intensity for line $i$ of phase $\alpha$ divided by the intensity for line $j$ of the internal standard $\mathrm{s}$. This ratio of the intensities gives an equation linear in the weight fraction of phase $\alpha$ :

$$
\frac{\mathrm{I}_{\mathrm{i} \alpha}}{\mathrm{I}_{\mathrm{js}}}=\mathrm{K}^{\prime} \frac{\mathrm{X}_{\alpha}}{\mathrm{X}_{\mathrm{s}}}
$$

Equation (3) permits the analysis of samples with the addition of an arbitrarily selected, but known, amount of internal standard. To establish a calibration curve, $\mathrm{X}_{\mathrm{s}} \mathrm{x}\left(\mathrm{I}_{\mathrm{i} \alpha} / \mathrm{I}_{\mathrm{j} s}\right)$ is plotted versus $\mathrm{X}_{\alpha}$. The slope of this straight line is $\mathrm{K}^{\prime}$. Note that $\mathrm{X}_{\alpha}$ represents the weight fraction of phase $\alpha$ in the mixture of sample plus internal standard. The weight fraction, $\mathrm{X}_{\alpha}^{\prime}$, of phase $\alpha$ in the original sample is given by

$$
\mathrm{X}_{\alpha}^{\prime}=\frac{\mathrm{X}_{\alpha}}{1-\mathrm{X}_{\mathrm{s}}}
$$

For routine analyses the weight fraction of the internal standard is often specified in the procedure. For example, a laboratory's quantitative XRD procedure may call for adding $0.1 \mathrm{gm}$ of internal standard to $0.9 \mathrm{gm}$ of sample. In such cases $\mathrm{X}_{\mathrm{s}}$ and $1-\mathrm{X}_{\mathrm{s}}$ are constants of the procedure and Equation (3) can be simplified to

$$
\frac{\mathrm{I}_{\mathrm{i} \alpha}}{\mathrm{I}_{\mathrm{js}}}=\mathrm{K}^{\prime \prime} \mathrm{X}_{\alpha}^{\prime}
$$

Use of a preestablished calibration curve defined by the slope $\mathrm{K}^{\prime \prime}$ yields the weight fraction of phase $\alpha$ in the original mixture. Note that $\mathrm{K}^{\prime \prime}$ is a function of $\mathrm{i}, \mathrm{j}, \alpha$, and s for a constant weight fraction of standard.

Systematic errors which vary with $\mathrm{X}_{\mathrm{s}}$ would not be detected in a calibration using Equation (5). Both microabsorption (Cline, 1988) and preferred orientation can be dependent on $\mathrm{X}_{\mathrm{s}}$. Thus, for improved reliability, use of several mixtures of the internal standard with a calibration sample, each with a different $\mathrm{X}_{\mathrm{s}}$, is recommended to determine $K^{\prime}$. The reduction of preferred orientation effects can also be achieved by measuring several diffraction lines from phase $\alpha$ and phase s. The use of multiple lines from each phase could be accommodated by independently determining $\mathrm{K}^{\prime}$ (or $\mathrm{K}^{\prime \prime}$ ) for each analyte line, internal-standard line pair. If three analyte lines and two standard lines were to be measured, a total of 6 calibration constants would be required ( $3 \times 2$ lines). A more general approach, which avoids introduction of multiple calibration constants, is to use the relative intensities, Irel, and the RIR. This more general equation is

$$
\frac{\mathrm{I}_{\mathrm{i} \alpha}}{\mathrm{I}_{\mathrm{j} \mathrm{s}}} \times \frac{\mathrm{I}_{\mathrm{j} s}^{\mathrm{rel}}}{\mathrm{I}_{\mathrm{i} \alpha}^{\mathrm{rel}}} \mathrm{x} \frac{\mathrm{X}_{\mathrm{s}}}{\mathrm{X}_{\alpha}}=\mathrm{K}=\mathrm{RIR}_{\alpha, \mathrm{s}}
$$

In Equation (6) $\mathrm{K}$ is a function of $\alpha$ and s but not $\mathrm{i}, \mathrm{j}$, or $\mathrm{X}_{\mathrm{s}}$. In the example above ( $3 \times 2$ lines) the RIR and five Irel values are required. However, since one line of the analyte and one of the internal standard can be assigned Irel $=100$, a total of only four calibration constants is required. An added bonus is that the Irel constants are readily understood by the user and are frequently used in other applications.
It should be noted, however, that the relative intensities in the PDF are seldom accurate enough for this application. The user should accurately determine the relative intensities in question or, more simply, allow calculation of them from the calibration sample measurements. Once this calibration constant RIR is determined it need not be redetermined before each analysis since variations in the incident beam intensity cancel in the $\mathrm{I}_{\mathrm{i} \alpha} / \mathrm{I}_{\mathrm{js}}$ ratio, just as $(\boldsymbol{\mu} / \boldsymbol{\rho})_{\mathrm{m}}$ is eliminated.

The slope $\mathrm{K}$ of the calibration curve defined by Equation (6) is a universal constant relating the scattering power of phase $\alpha$ to that of phase s. $\mathrm{K}$ is known as the Reference Intensity Ratio or RIR (Hubbard, Evans \& Smith, 1976; Chung, 1975). To specify which phase and which internal standard, a pair of subscripts $(\alpha, s)$ are used. Requirements on measurement procedures to obtain accurate RIR values are: (1) freedom from preferred orientation and microabsorption; (2) constant irradiated volume of sample independent of scattering angle; (3) correction for monochromator polarization effects; and (4) use of integrated intensities. Of course the calibration sample must be well characterized chemically and represent the chemistry of the analyte. Noninterfering second phases, including amorphous phases, may be present in a calibration sample; however, the concentration of $\alpha$ and s must be known exactly. The fact that the RIR, when properly measured, is a universal constant means that RIR values determined in one laboratory can be used in any other laboratory which chooses to meet the four requirements.

If calibration and analytical data are to be collected on a single instrument, then the requirements for measuring the $\mathrm{K}$ value can be reduced to: (1) reproducible orientation and microabsorption effects for calibration standard and unknown; (2) reproducible irradiated volume of sample for each $2 \theta$; (3) measurement of peak or integrated intensities in a reproducible way. In this case $\mathrm{K}$ is not a universal constant, but rather a constant for the sample mounting and data collection method.

It is clear from the general definition of the Reference Intensity Ratio given in Equation (6) that this value can be measured for any substance using any convenient diffraction line of both phase $\alpha$ and the internal standard when the two substances are mixed in a known weight ratio. The RIR, when accurately measured, is a true constant and allows comparison of the absolute diffraction line intensities of one material to another. It also enables quantitative phase analysis in a number of convenient and useful ways.

\section{I/I corundum}

A particularly important case is when corundum is chosen as the internal standard reference material and integrated intensities are used. In this case the RIR is referred to as "I over I corundum" or more simply, I/I $\mathrm{I}_{\mathrm{c}}$. The PDF contains $I / I_{c}$ values given for over 2500 phases which are published in the PDF Search Manuals and on the corresponding PDF cards. Nearly all have been determined using peak height ratios as an approximation for integrated intensity ratios or are calculated using reported atomic position and thermal parameters. Although the experimental values are subject to measurement and sample related errors (Cline, 1988), they are useful in obtaining a quick 
estimate of the concentration of the phases in an unknown. However, unless the relative intensity and the RIRs are carefully determined by the user, the analysis based on tabulated $I / I_{c}$ values should be referred to as semiquantitative.

\section{Measurement of $I / I_{c}$}

By definition $\left(\mathrm{I} / \mathrm{I}_{\mathrm{c}}\right)_{\alpha}$ is the ratio of the integrated intensities for $\mathrm{CuK}_{\alpha}$ radiation of the strongest line (Irel $=100$ ) of phase $\alpha$ to the strongest line of corundum for a 1:1 mixture by weight. Using this definition it is easy 'to quickly measure $\mathrm{I} / \mathrm{I}_{\mathrm{c}}$. One makes the $1: 1$ mixture, measures the integrated intensities and directly calculates $\mathrm{I} / \mathrm{I}_{\mathrm{c}}$. For even quicker results the ratio of peak height intensities can be used to approximate the ratio of integrated intensities. This is best used when the grain size is small and all the line breadths are approximately equal.

This simple 2-line procedure is quick but suffers from several drawbacks. First, preferred orientation often affects the strong lines unless careful mounting methods are employed. Other problems include extinction and inhomogeneity of mixing. For greater accuracy Hubbard and Smith (1977) have recommended using multiple lines from both the sample and the reference phases and using multiple sample mounts. Such an approach often reveals that preferred orientation is present and provides realistic measurement of the reproductibility in the measurement of $I / I_{c}$.

\section{Quantitative Analysis with $I / I_{c}$}

Selected methods of employing $\mathrm{I} / \mathrm{I}_{\mathrm{c}}$ and Reference Intensity Ratios are discussed in this section.

Case 1. Analysis when a known amount of corundum is added to a general unknown:

In this case corundum is the internal standard. If we rearrange Equation (6) we get

$$
\mathrm{X}_{\alpha}=\frac{\mathrm{I}_{\mathrm{i} \alpha}}{\mathrm{I}_{\mathrm{jc}}} \times \frac{\mathrm{I}_{\mathrm{jc}}^{\mathrm{rel}}}{\mathrm{I}_{\mathrm{i} \alpha}^{\mathrm{rel}}} \times \frac{\mathrm{X}_{\mathrm{c}}}{\mathrm{RIR}_{\alpha, \mathrm{c}}}=\frac{\mathrm{I}_{\mathrm{i} \alpha}}{\mathrm{I}_{\mathrm{jc}}} \times \frac{\mathrm{I}_{\mathrm{jc}}^{\mathrm{rel}}}{\mathrm{I}_{\mathrm{i} \alpha}^{\mathrm{rel}}} \times \frac{\mathrm{X}_{\mathrm{c}}}{\left(\mathrm{I} / \mathrm{I}_{\mathrm{c}}\right)_{\alpha}}
$$

The use of an $\left(I / I_{c}\right)_{\alpha}$ value from the PDF allows us to avoid preparing our own standards to determine the calibration constant. When the qualitative analysis of one or more phases with known $I / I_{c} s$ is desired we simply have to add a known amount of corundum to the sample and use Equation (7). Analysis is possible for $\mathrm{X}_{\alpha}$ using any combination of diffraction lines from phase $\alpha$ and corundum. This use of $\mathrm{RIR}_{\alpha, \mathrm{c}}$ constants (i.e., $\mathrm{I}_{\mathrm{c}} \mathrm{I}_{\mathrm{c}}$ values) and possibly Irel values from the PDF should be considered as semiquantitative due to the unspecified errors in the constants. However, if Irel and RIR values are accurately known and other systematic errors are absent Equation (7) can yield accurate quantitative results.

Case 2. Analysis when a known amount of an internal standard is added to a sample:

From Equation (6) it is readily shown that

$$
\operatorname{RIR}_{\alpha, \mathrm{s}}=\frac{\operatorname{RIR}_{\alpha, \mathrm{c}}}{\operatorname{RIR}_{\mathrm{s}, \mathrm{c}}}=\frac{\left(\mathrm{I} / \mathrm{I}_{\mathrm{c}}\right)_{\alpha}}{\left(\mathrm{I} / \mathrm{I}_{\mathrm{c}}\right)_{\mathrm{s}}}
$$

Hence, we can combine $I / I_{c}$ values for phases $\alpha$ and $s$ to obtain the RIR for phase $\alpha$ relative to phase s. Taking $s$ to be an internal standard and substituting Equation (8) into Equation (6) and rearranging we have

$$
\mathrm{X}_{\alpha}=\frac{\mathrm{I}_{\mathrm{i} \alpha}}{\mathrm{I}_{\mathrm{j} \mathrm{s}}} \times \frac{\mathrm{I}_{\mathrm{js}}^{\mathrm{rel}}}{\mathrm{I}_{\mathrm{i} \alpha}^{\mathrm{rel}}} \times \frac{\mathrm{RIR}_{\mathrm{s}, \mathrm{c}}}{\mathrm{RIR}_{\alpha, \mathrm{c}}} \mathrm{X}_{\mathrm{s}}
$$

This equation is quite general and allows for the analysis of any crystalline phase with a known RIR in an unknown mixture by the addition of a known amount of any internal standard. However, we are using four constants from the literature

$\left(\mathrm{I}_{\mathrm{i} \alpha}^{\mathrm{rel}}, \mathrm{I}_{\mathrm{js}}^{\mathrm{rel}}, \mathrm{RIR}_{\mathrm{s}, \mathrm{c}}\right.$, and $\left.\mathrm{RIR}_{\alpha, \mathrm{c}}\right)$, each of which may contain significant error.

Case 3. Analysis of weight fraction ratios using RIRs:

Equation (9) is valid even for complex mixtures which contain unidentified phases, amorphous phases, or identified phases with unknown RIRs. The ratio of the weight fractions of any two phases whose RIRs are known may always be computed by choosing one as $\mathrm{X}_{\alpha}$ and the other as $\mathrm{X}_{\mathrm{s}}$.

That is

$$
\mathrm{X}_{\alpha} / \mathrm{X}_{\beta}=\frac{\mathrm{I}_{\mathrm{i} \alpha}}{\mathrm{I}_{\mathrm{j} \beta}} \times \frac{\mathrm{I}_{\mathrm{j} \beta}^{\mathrm{rel}}}{\mathrm{I}_{\mathrm{i} \alpha}^{\mathrm{rel}}} \times \frac{\mathrm{RIR}_{\beta, \mathrm{c}}}{\mathrm{RIR}_{\alpha, \mathrm{c}}}
$$

Case 4. Analysis of a mixture of identified phases without the addition of an internal standard:

As Chung (1975) pointed out, if all the phases in a mixture are identified and the RIR value is known for each phase, the additional equation

$$
\sum_{\mathrm{k}=1}^{\mathrm{n}} \mathrm{X}_{\mathrm{k}}=1
$$

where $\mathrm{n}=$ the number of phases in the mixture, permits analysis without adding any standard to the unknown specimen. This is seen by arbitrarily choosing one of the phases in the mixture as s and then using Equation (10) to solve for the ratio of the weight fraction of each phase to the weight fraction of s (i.e., $\mathrm{X}_{\alpha} / \mathrm{X}_{\mathrm{s}}, \mathrm{X}_{\beta} / \mathrm{X}_{\mathrm{s}}$, etc.). For $\mathrm{n}$ phases in the mixture this produces $\mathrm{n}-1$ ratios. The relationship given in Equation (11) (i.e., $\mathrm{X}_{\mathrm{s}}+\mathrm{X}_{\alpha}$ $\left.+\mathrm{X}_{\beta}+\ldots=1\right)$ allows the writing of the $\mathrm{n}^{\text {th }}$ equation. Chung (1975) has shown that this system of $n$ linear equations can be solved to produce the weight fractions of the $n$ unknowns as shown in Equation 12. It is important to note that the presence of any amorphous phase invalidates the use of Equation (12).

$$
\mathrm{X}_{\alpha}=\frac{\mathrm{I}_{\mathrm{i} \alpha}}{\operatorname{RIR}_{\alpha} \times \mathrm{I}_{\mathrm{i} \alpha}^{\mathrm{rel}}} \times\left[\frac{1}{\sum_{\mathrm{j}=1} \frac{\mathrm{I}_{\mathrm{j} \alpha}}{\mathrm{RIR}_{\mathrm{j}} \cdot \mathrm{I}_{\mathrm{j} \alpha}^{\mathrm{rel}}}}\right]
$$

Computer Code

Program QUANT in the NBS*QUANT84 package for quantitative XRD analysis (Snyder, Hubbard and Panagiotopoulos, 1981, 1982) implements the general Equation 6. Minor extensions to the program would be required to implement cases 3 and 4 above. If the RIR values are 
known from another source they may be directly used along with the relative intensities. However, for improved accuracy a better procedure is to add an internal standard to pure specimens of each phase of interest in the mixture and produce a series of calibration data files using integrated intensities which can be analyzed by the program RUNFIL. Program RUNFIL will compute the relative intensities of the measured lines in each phase and the Reference Intensity Ratios.

\section{Line Overlap}

Line overlap can be allowed for following the method of Copeland and Bragg (1958) along with the application of chemical constraints. For m measured intensity regions, the Copeland/Bragg equations for multicomponent quantitative analysis with possible line superposition form a system of $\mathrm{m}$ simultaneous linear equations. Each equation is of the form

$$
\begin{aligned}
I_{\mathrm{i}} / \mathrm{I}_{\mathrm{n}}= & \left(\mathrm{C}_{\mathrm{i} 1} / \mathrm{C}_{\mathrm{ns}}\right) \frac{\mathrm{X}_{1}}{\mathrm{X}_{\mathrm{s}}}+\left(\mathrm{C}_{\mathrm{i} 2} / \mathrm{C}_{\mathrm{ns}}\right) \frac{\mathrm{X}_{2}}{\mathrm{X}_{\mathrm{s}}} \ldots+ \\
& \left(\mathrm{C}_{\mathrm{ik}} / \mathrm{C}_{\mathrm{ns}}\right) \frac{\mathrm{X}_{\mathrm{k}}}{\mathrm{X}_{\mathrm{s}}}+\ldots \epsilon_{\mathrm{i}}
\end{aligned}
$$

where $\mathrm{I}_{\mathrm{i}}$ is the intensity of the $\mathrm{i}^{\text {th }}$ line from the mixture of $P$ phases $(1<\mathrm{i} \leq \mathrm{m}$ and $1 \leq \mathrm{k} \leq \mathrm{P}) ; \mathrm{I}_{\mathrm{n}}$ is the intensity of a resolved line of the internal standard $\mathrm{s}$; $\mathrm{X}_{\mathrm{k}}$ is the weight fraction of phase $\mathrm{k}$ in the mixture of sample plus internal standard; $\mathrm{C}_{\mathrm{ik}}$ is a constant including the mass absorption coefficient of the mixture and the diffraction intensity of the $\mathrm{i}^{\text {th }}$ line of phase $\mathrm{k}$; and $\epsilon_{\mathrm{i}}$ is an error term. From $\mathrm{X}_{\mathrm{k}}$ and $X_{s}$ the weight fractions $X_{k}$ of the original sample can be computed by

$$
\mathrm{X}_{\mathrm{k}}=\frac{\mathrm{X}_{\mathrm{k}}}{1-\mathrm{X}_{\mathrm{s}}}
$$

Thus, to allow for mixtures of the sample with addition of differing concentrations of internal standard, the matrix coefficients, set in program QUANT, are:

$$
\frac{\mathrm{C}_{\mathrm{ik}}}{\mathrm{C}_{\mathrm{ns}}} \times \frac{1}{\mathrm{X}_{\mathrm{s}}} \times \frac{1}{\left(1-\mathrm{X}_{\mathrm{s}}\right)}
$$

All these matrix coefficients must be known for one to determine the values $\mathrm{X}_{\mathrm{k}}$. The $\mathrm{C}_{\mathrm{ik}} / \mathrm{C}_{\mathrm{ns}}$ ratios are given by the product of the ratio of the corresponding relative intensities of the pure phases and the reference intensity ratio, that is

$$
\mathrm{C}_{\mathrm{ik}} / \mathrm{C}_{\mathrm{ns}}=\left(\mathrm{I}_{\mathrm{ik}}^{\mathrm{rel}} / \mathrm{I}_{\mathrm{ns}}^{\mathrm{rel}}\right) \times \mathrm{RIR}_{\mathrm{k}, \mathrm{s}}
$$

From this expression for $\mathrm{C}_{\mathrm{ik}} / \mathrm{C}_{\mathrm{ns}}$ the Copeland and Bragg matrix elements can readily be obtained from Irel and RIR tabulations, calculations, and/or experimental measurements. If phase $\mathrm{k}$ has zero intensity at pattern line $\mathrm{i}$ then $I_{\mathrm{ik}}^{\text {rel }}=0$ and thus $\mathrm{C}_{\mathrm{ik}} / \mathrm{C}_{\mathrm{ns}}=0$. In general $\mathrm{m}>\mathrm{P}$ and the system of equations can be solved by least squares techniques where the sum of $\epsilon_{\mathrm{i}}^{2}$ is minimized.

\section{Constraints}

Quantitative elemental data, obtained from X-ray fluorescence analysis for example, can be added to the system of equations in diffraction intensity (Equation 13) without increasing the number of unknowns. Coupling both the elemental and diffraction data should result in more accurate quantitative analysis of multicomponent mixtures. More importantly, estimates of the standard deviation of the results will be more accurate. To invoke this special feature, chemical composition for each phase to be analyzed must be included, and all phases containing the quantified element(s) must be included in the XRD phase analysis. The equation relating weight fraction in each phase to the overall weight fraction, expressed as a percentage for element $A$ is

$$
\begin{aligned}
(\% \mathrm{~A})_{\text {mixture }}= & (\% \mathrm{~A})_{1} \mathrm{X}_{1}+(\% \mathrm{~A})_{2} \quad \mathrm{X}_{2} \ldots \\
& (\% \mathrm{~A})_{\mathrm{k}} \mathrm{X}_{\mathrm{k}}+\epsilon
\end{aligned}
$$

where $(\% \mathrm{~A})_{\mathrm{i}}$ is the weight percent of element $\mathrm{A}$ in phase i. Such linear equations in $\mathrm{X}_{1} \ldots \mathrm{X}_{2}$ can be combined with Equation (13). The expanded system of equations is again solved by least-squares techniques. Both the line overlap and chemical analysis features have been included in the NBS*QUANT84 package. In a similar manner an equation for the mass absorption coefficient of the mixture could be used either as a constraint or an additional "observation" similar to Equation (17).

\section{Summary}

The internal standard method has been shown to be particularly general, and the calibration constants can be expressed in terms of the Reference Intensity Ratio. When the internal standard is corundum the RIR value is also called $I / I_{C}$. Tabulation or calculation of $I / I_{c}$ values permits semiquantitative analysis by routine data collection methods.

\section{References}

Alexander, L. E. and Klug, H. P. (1948). Anal. Chem. 20, 886.

Chung, F. H. (1975). Quantitative Interpretation of X-Ray Diffraction Patterns. III. Simultaneous Determination of a Set of Reference Intensities. J. Appl. Crystallogr. 8, 17.

Clark, G. L. \& Reynolds, D. H. (1936). Ind. Eng. Chem. Anal. Ed. 8, 36

Cline, J. (1988). An Experimental Verification of A. W. Brindley's Microabsorption Theory. In preparation.

Copeland, L. E. and Bragg, R. H. (1958). Anal. Chem. 30, 196.

Hubbard, C. R., Evans, E. H. \& Smith, D. K. (1976). The Reference Intensity Ratio, $\mathrm{I} / \mathrm{I}_{\mathrm{c}}$, for Computer Simulated Patterns. J. Appl. Crystallogr. 9, 169.

Hubbard, C. R. \& Smith, D. K. (1977). In Adv. X-Ray Anal., ed H. F. McMurdie, C. S. Barrett, J. B. Newkirk \& C. O. Ruud, 20, 63. New York: Plenum.

Klug, H. P. \& Alexander, L. E. (1974). X-Ray Diffraction Procedures. 2nd ed. (a)549-553, (b)365-368. New York: J. Wiley and Sons.

Lennox, D. H. (1957). Anal. Chem. 29, 767

Navias, A. L. (1925). J. Am. Ceram. Soc. 8, 296.

Powder Diffraction File (1987). Swarthmore, PA: International Centre for Diffraction Data.

Smith, S. T., Snyder, R. L. \& Brownell, W. E. (1979). The Quantitative Analysis of Devonian Shales. In Adv. X-Ray Anal., ed. G. J. McCarthy, C. S. Barrett, D. E. Leyden, J. B. Newkirk \& C. O. Ruud, 22, 181. New York: Plenum.

Snyder, R. L., Hubbard, C. R. \& Panagiotopoulos, N. C. (1981) AUTO: A Real Time Diffractometer Control System. NBSIR 81-2229. U. S. Dept. of Commerce, Natl. Bur. Stand. Gaithersburg, MD 20899

Snyder, R. L., Hubbard, C. R. \& Panagiotopoulos, N. C. (1982). A Second Generation Automated Powder Diffraction Control System. In Adv. X-Ray Anal., ed. J. C. Russ, C. S. Barrett, P. K. Predecki $\&$ D. E. Leyden, 25, 245. New York: Plenum.

Visser, J. W. \& Wolff, P. M. de (1964). Absolute Intensities. Report 641.109, Technisch Physische Dienst, Delft, Netherlands. 